\title{
SUPPORTING INFORMATION: Evaporation Dynamics in Buried Nanochannels with Micropores
}

\author{
Sajag Poudel, An Zou, Shalabh C. Maroo* \\ Department of Mechanical \& Aerospace Engineering, Syracuse University \\ Syracuse, NY 13210 \\ *Corresponding Author E-mail: scmaroo@syr.edu
}

Number of pages: 6

Number of figures: 4

Number of schemes: 5

Number of tables: 1

Table of Contents

Section

Tltle

Page

S1 Nanofabrication

S2

S2 Nanochannels sample characterization.

S3

S3 Droplet wicking experiments.

S3

S4 Computational Fluid Dynamics (CFD) Simulation Details.

S4

S5 Analytical Models.

S5 


\section{S1. Nanofabrication}

The buried nanochannels were obtained by etching patterned buried sacrificial metal layers $(\mathrm{Cr}+$ $\mathrm{Cu}$ in our case). Thus, the channel geometry is determined by the pattern dimensions. The channel width was designed as $5 \mu \mathrm{m}$ and $10 \mu \mathrm{m}$ for the two samples with the ratio of spacing to width as 1 . Due to the defect during fabrication process, the actual width and spacing were 4.5 $\mu \mathrm{m}$ and $5.7 \mu \mathrm{m}$ for S5 sample, and these were the dimensions used in CFD simulations (see Table 1 of manuscript). The channel height is determined by the thickness of the metal layers, which were deposited using e-beam evaporator. Due to the low pressure during the deposition, most atoms were deposited on the substrate without intervening collisions with other gas molecules as the distance between the source and substrate is smaller than the mean free path of the particles. Thus, excellent uniformity can be achieved [1]. Figures S1-a and S1-b show the atomic force microscopy AFM image of the patterned metal layers and the corresponding height profile. The mean roughness $\left(R_{a}\right)$ is $\sim 3 \mathrm{~nm}$ with a thickness of $728 \mathrm{~nm}$. The uniformity across the entire substrate was confirmed by the repeatable results from AFM images at four different locations on the substrate. Figures S1-c and S1-d show the AFM image of the final sample (after sacrificial layers were etched). The height was preserved. The extra surface roughness $\left(R_{a}\right.$ is $\sim 10$ $\mathrm{nm}$ ) is brought during the plasma enhanced chemical vapor deposition (PECVD) of $\mathrm{SiO}_{2}$, which would not affect the top surface of nanochannels. Further details on the steps of nanofabrication are available in our recent articles [2, 3].

(a)
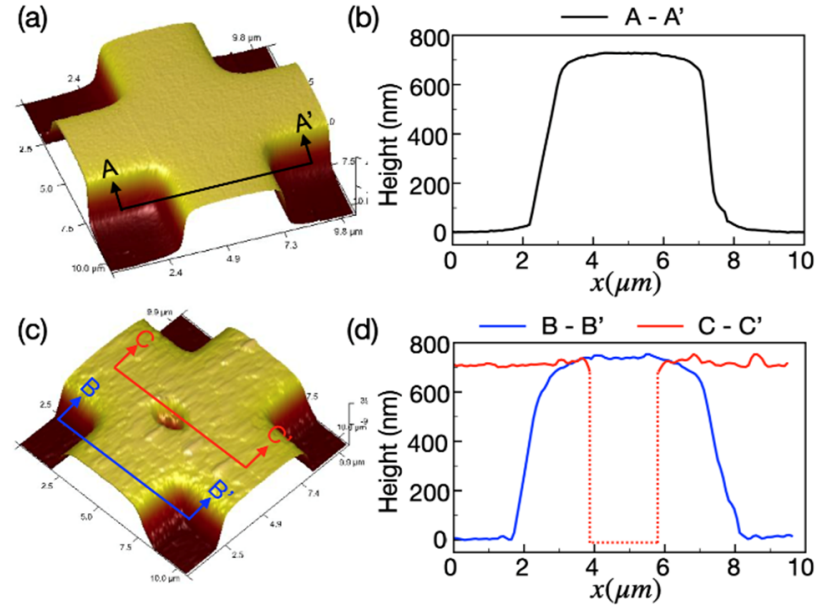

Figure S1: AFM Images of nanochannels sample and corresponding height profiles. (a) AFM image of a patterned metal layers, (b) Height profile of the deposition, (c) AFM image of a unit cell of nanochannels sample obtained after etching of the sacrificial layers, (d) Height profile across the nanochannel width and the micropore of the final sample. The solid lines in the plot (b, d) represent the actual AFM data while the dotted line in (d) designates the expected data across the micropore.

The channel height is designed based on the following three limitations: (1) the height is limited by the maximum thickness of the sacrificial layers, which is around $1 \mu \mathrm{m}$ due to the film stress, (2) the other factors affecting wicking in channels should be excluded, such as electroviscous effect, disjoining effect, etc. which come into the picture around $\sim 100 \mathrm{~nm}$ and less, and (3) the wicking distance should be large enough to conduct analysis with minimum errors, which prefers higher channels under the prior two limitations. Thus, the channel height was chosen in the 
submicron range to be $\sim 700 \mathrm{~nm}$, and it turned out to be $728 \mathrm{~nm}$ after the nano-fabrication processes. The same height of $728 \mathrm{~nm}$ is used in the geometry for CFD simulations.

\section{S2. Nanochannels Sample Characterization}

The fabricated nanochannel samples are characterized using contact angle of a sessile droplet placed on the top surface. The goniometer used is VCA Optima of AST Products Inc., which has a repeatability of $1^{\circ}$ and an accuracy of $0.5^{\circ}$. The Goniometer images and corresponding contact angles of each nanochannel sample as well as the bare sample are shown in Figure S2. Due to water wicking into the channels (Wenzel state), the nanochannels sample are hydrophilic.

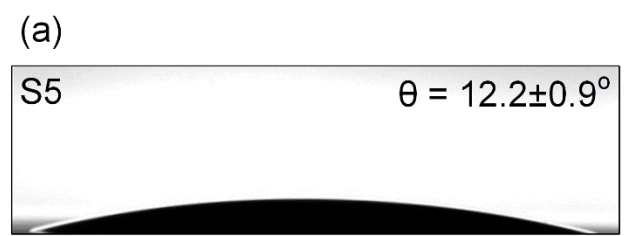

(b)

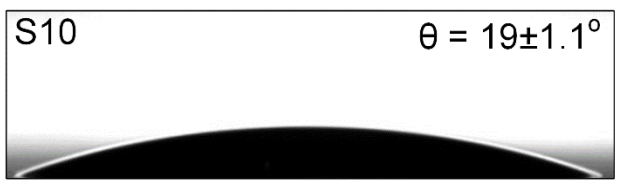

(c)

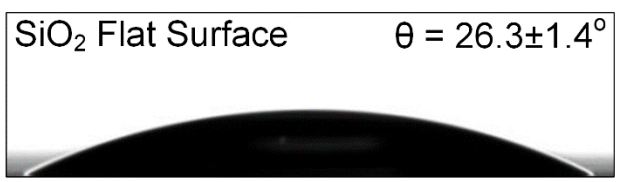

Figure S2: Contact angle measurement using Goniometer images of sessile droplet sitting on the nanochannels sample (a) S5 and (b) $\mathrm{S} 10$, and (c) flat surface of $\mathrm{SiO}_{2}$ (bare sample).

\section{S3. Droplet Wicking Experiments}

The fabricated nanochannels sample is placed on a stage capable to traverse vertically as shown in Figure S3. A DI water droplet of specified volume is fed through a syringe controlled by an automatic pump. A high-speed camera is used for the time-resolved feature of the liquid spreading as well as receding menisci (wicking front) inside the nanochannels. The receding menisci is the resultant of the evaporation in the interfaces at nanochannels and micropores. The receding phase occurs quickly and thus we need a high-speed camera to capture multiple frames. The frame rate used is $50 \mathrm{fps}$. The model of the camera used is Phantom V611. The automatic pump used for sessile droplet generation has the least count of $0.1 \mu \mathrm{l}$ and the error of $<0.35 \%$. The experiments were conducted at relative humidity $\mathrm{RH}=44.2 \pm 1.3 \%$. 


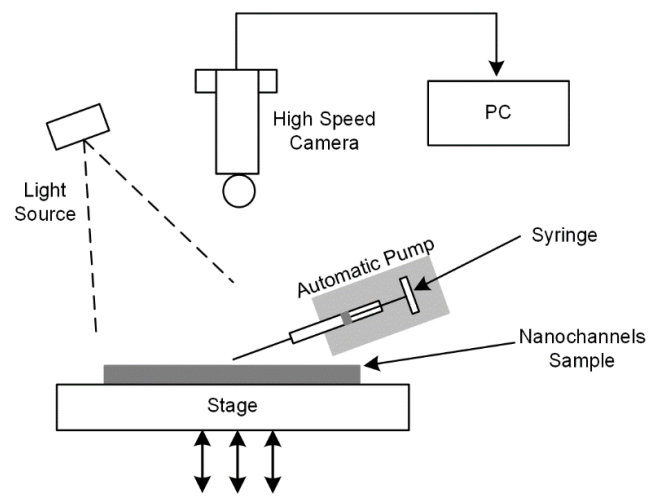

Figure S3: Experimental setup for droplet wicking test on nanochannels sample

\section{S4. Computational Fluid Dynamics (CFD) Simulation Details}

\section{Experimental validation of the CFD results}

CFD simulations are performed in the nanochannels geometry consistent with the actual experimental samples. Multiphase volume of fluids for laminar flow simulations is evoked and the discretized form of the governing equations are solved. Evaporation is evoked at the liquid-air interface by tracking the volume fraction of liquid phase $\sim 0.5$ using a user defined function (udf). Liquid volume fraction $=0.5$ represents the liquid-air interface in the computational domain. However, in certain instance, there might not be any computational cells with the liquid volume fraction exactly equal to 0.5 . Thus, the udf is finally assigned to evoke evaporation in a range of liquid volume fraction $0.48-0.52$. Different meshes are used to achieve grid independence and, by utilizing the optimum mesh, the obtained results of liquid spreading (wicking radius $R_{w}$ ) from CFD is compared with the experimental wicking results on the same nanochannels sample geometry. The CFD results show an excellent agreement with the experiments as shown in Figure S4. Additional details of similar numerical simulations are also available in our recent article [3]. The run time for carrying out one complete simulation was $\sim 48$ hrs using a 4-core i7-4790 processor of $3.6 \mathrm{GHz}$ and $32 \mathrm{~GB}$ RAM.

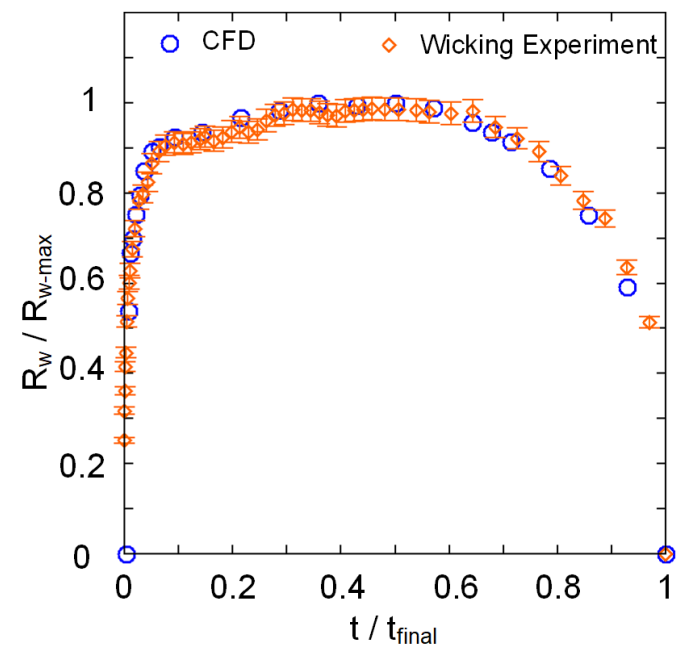

Figure S4: Experimental validation of CFD results using wicking experiments for sample S5. 


\section{Parameters for non-dimensional time $t^{*}$}

In Eq. 1 of the manuscript, a new non-dimensional parameter for time is defined. For the same $t^{*}$, the associated time instants at which spherical cap completely wicks in $\left(t_{n o-s p}\right)$ and the final time instant where all liquid inside nanochannels completely evaporate $\left(t_{\text {final }}\right)$ are shown in Table S1. The computational time step was taken as $10^{-8}$ seconds.

Table S1: Parameters associated with non-dimensional time $t^{\star}$

\begin{tabular}{|l|c|c|}
\hline \multicolumn{1}{|c|}{ Case } & $\begin{array}{c}t=t_{\text {no-sp }} \\
\left(\mathrm{t}^{*}=0\right)\end{array}$ & $\begin{array}{c}t=t_{\text {final }} \\
\left(\mathrm{t}^{*}=1\right)\end{array}$ \\
\hline S5-CFD-All & $750 \mu \mathrm{s}$ & $965 \mu \mathrm{s}$ \\
\hline S5-CFD-Ch,Po & $1060 \mu \mathrm{s}$ & $1405 \mu \mathrm{s}$ \\
\hline S10-CFD-All & $1530 \mu \mathrm{s}$ & $1925 \mu \mathrm{s}$ \\
\hline S10-CFD-Ch,Po & $1850 \mu \mathrm{s}$ & $2385 \mu \mathrm{s}$ \\
\hline
\end{tabular}

\section{S5. Analytical Models}

From the experimental and numerical study of wicking and evaporation, we have also compared our results with those obtained from different analytical models which is explained next.

\section{Capillary pressure from thermodynamic definition}

The analytical value of the capillary pressure presented in Figure $3(d)$ of the manuscript is calculated from the thermodynamics definition $[2,4]$ which is based on the nanochannels unit cell (height $\mathrm{H}$ and width $\mathrm{W}$ of the nanochannels). The corresponding equation is as follows [2]:

$\mathrm{P}_{\text {cap }-\mathrm{td}}=\frac{\Delta \mathrm{E}}{\Delta \mathrm{V}}=\frac{\gamma \mathrm{r}_{\mathrm{f}} \cos \theta_{\mathrm{c}}\left[2 *\left\{(\mathrm{~W}+\mathrm{S})^{2}-\mathrm{W}^{2}\right\}+4 \mathrm{WH}\right]}{\mathrm{H}\left((\mathrm{S}+\mathrm{W})^{2}-\mathrm{S}^{2}\right)}$

where, $\mathrm{P}_{\text {cap-td }}$ is the change is surface energy $\Delta \mathrm{E}$ to volume of a unit cell of nanochannels $\Delta \mathrm{V}, \gamma$ is surface tension, $r_{f}$ is the roughness factor for the nanochannels wall which is close to 1 (from AFM images in Figure S1), $\theta_{c}=26^{\circ}$ is the intrinsic contact angle of liquid (deinoized water) on $\mathrm{SiO}_{2}$ surface (see Figure S2).

\section{Calculation of permeability and viscous resistance}

For the given cross-connected nanochannels sample, the scale and geometry of channels are well defined and porosity is precisely known i.e. $\varepsilon=0.70$ and 0.73 for samples S5 and S10, respectively. This helps us to replicate the geometry of the sample in the CFD model. The nanochannels width, spacing, height and micropore diameter in CFD model are consistent with that used in the experiments, thus we use the same porosity $(\varepsilon)$ in CFD.

Additionally, for such uniform micro/nano structures, permeability is calculated by using an analytical equation developed by Sangani and Acrivos [5] as follows:

$\alpha^{-2}=l^{2} \frac{\ln c^{-1 / 2}-0.738+c-0.887 c^{2}+2.038 c^{3}+o\left(c^{4}\right)}{4 \pi}$

where, $\alpha^{-2}$ is permeability, $c$ is the solid fraction of sample such that $c=1-\varepsilon$ and $\varepsilon$ is porosity, $l$ is the length of a unit cell of nanochannels i.e. $l=W+S$. 
Next, the analytical value of viscous resistance $\mathrm{K}_{\mathrm{vr}}$-Analyt (Figure 4 of manuscript) is calculated as the ratio of $d P / d x$ to $u_{\text {mean. }}$. This particular ratio is in turn derived from the Brinkman's equation [4] as follows:

$\mu \frac{d^{2} u}{d y^{2}}-\varepsilon \frac{d P}{d x}-\mu \alpha^{2} \varepsilon u=0$

In the above equation, $\alpha^{-2}$ is permeability calculated from Eq. S2, and $\mathrm{u}$ is the wicking velocity. Eq. S3 is solved for the nanochannels geometry with no-slip boundary conditions at walls followed by integration in the limit $y=-H / 2$ to $y=H / 2$ where $H=$ height of nanochannels [2] which results into the ratio of $d P / d x$ to $u_{\text {mean }}$.

\section{References}

1. Madou, M.J., Fundamentals of microfabrication : the science of miniaturization. 2001, Boca Raton, FL: CRC Press.

2. Poudel, S., A. Zou, and S.C. Maroo, Wicking in Cross-Connected Buried Nanochannels. The Journal of Physical Chemistry C, 2019. 123(38): p. 23529-23534.

3. Zou, A., S. Poudel, S.P. Raut, and S.C. Maroo, Pool Boiling Coupled with Nanoscale Evaporation Using Buried Nanochannels. Langmuir, 2019. 35(39): p. 12689-12693.

4. Xiao, R., R. Enright, and E.N. Wang, Prediction and Optimization of Liquid Propagation in Micropillar Arrays. Langmuir, 2010. 26(19): p. 15070-15075.

5. Sangani, A.S. and A. Acrivos, Slow flow past periodic arrays of cylinders with application to heat transfer. International Journal of Multiphase Flow, 1982. 8(3): p. 193-206. 